\author{
By: Rasheda Nyeem, M.D.*
}

In 1967 Baruch Blumberg and Gajdusek received Nobel prize in recognition of the finding of a "new antigen" in the blood of an Australian Aborigine, which was found to be associated with viral Hepatitis. Remarkable advances have been made in the field of viral hepatitis in the last sixteen years. This flooding of new information over a relatively short period of time appears, at times, to be rather confusing and complex. In this paper, I will discuss the types, etiology, clinical presentation, diagnosis, prophylaxis and the most current intervention namely vaccination types of hepatitis.

The term "viral hepatitis" exclusively refers to primary hepatic infection caused by well defined agents. These are hepatitis A virus; hepatitis B virus and Non A Non B viruses. Other viruses that may cause hepatitis are uncommon and not considered primary liver pathogens. These are cytomegaloviruses (CMV), Epstein Barr (EB), Herpes Simplex (HS), varicella-zoster and rubella viruses.

\section{Type A Hepatitis}

Hippocrates described Type A like disease about 2000 years ago. Epidemic and sporadic cases of this disease have been reported over the centuries. The virus of hepatitis A, previously known as "infectious hepatitis" was isolated in 1973 by Feinstone and his colleagues,' from the stool specimen of an acute hepatitis patient. Hepatitis A virus (HAV) is a small 27 nanometer ( $\mathrm{nm}$ ) RNA virus belonging to RNA group. The virus can be isolated one or two weeks before, and for a week or more after the onset of the clinical illness. Hepatitis A antibodies (anti HA) appear early with infection and remain detectable for life. Initially, these antibodies are of IGM type but within a few weeks are replaced by IGS antibodies. Acute hepatitis A is typically self limited with less than $0.1 \%$ fatality rate.

Progression to chronic hepatitis never occurs and there is no chronic carrier state, clinically, it is indistinguishable from acute hepatitis B. Fever, malaise, anorexia, nausea and abdominal discomfort are the usual presenting symptoms. Jaundice follows within a few days.

\footnotetext{
"Executive Medical Director, North East Florida State Hospital.

(Article submitted for publication on August 1, 1983.)
}

Diagnosis is established by a four-fold or greater increase in specific antibodies to $\mathrm{HAV}$, by radio immunoassay, or by the demonstration of virus in stool or the presence of specific IGM antibodies. In contrast to hepatitis B, the incubation period is short, lasting 15-45 days.

\section{Type B Hepatitis}

The causative agent for type $B$ hepatitis, hepatitis $B$ virus (HBV) was first visualized by Dane and his colleagues in 1970 , by electron microscopy, in sera of patients with hepatitis and is frequently referred as Dane Particle. HBV is a $42 \mathrm{~nm}$ DNA virus that belongs to a new class; called "Hepadna Viruses."2 It consists of a central core $27 \mathrm{~nm}$ in diameter surrounded by an outer surface coat. The central core contains the viral genetic material, (circular DNA) which is double stranded in $70 \%$ of its length and single stranded for $30 \%$. The outer coat is made up of protein, polysaccharide and lipids and is found in excess of Dane Particle.

The antigen antibody systems associated with $\mathrm{HBV}$ are "hepatitis B surface antigen" $\left(\mathrm{HB}_{\mathrm{s}} \mathrm{Ag}\right)$; core antigen $\left(\mathrm{HB}_{\mathrm{c}} \mathrm{Ag}\right)$ and $\mathrm{e}$ antigen $\left(\mathrm{HB}_{\mathrm{e}} \mathrm{Ag}\right)$. $\mathrm{HB}_{\mathrm{s}} \mathrm{Ag}$ is associated with viral surface coat. It signifies active $\mathrm{HB}$ infection. $\mathrm{HB}_{\mathrm{c}} \mathrm{Ag}$ is associated with viral core and is normally not detected in serum although it can be demonstrated in infected liver tissues. Antibodies to the above antigens have specific significance. Antibodies surface antigen $\left(\right.$ Anti $\mathrm{HB}_{\mathrm{s}}$ ) indicate a previous exposure and relative immunity to $\mathrm{HBV}$. Antibodies to core antigen are found in chronic B carriers and indicate active viral replication. e antigen has been identified as a soluble antigen, probably a component of nuclear capsid. This antigen appears to be a reliable and sensitive serological marker for the presence of high levels of viruses and thus a high degree of infectivity.

\section{Clinical Course}

The incubation period ranges from 40-180 days, and averages to 75 days. This variation is related to the inoculative dose of the virus, mode of transmission as well as host factors. Majority of patients who develop acute hepatitis recover uneventfully. 1-3\% develop fulminant disease and about $10 \%$ of patients develop chronic persistent hepatitis lasting for 3 to 6 months, with or without eventual cirrhosis. 
This last group indicates presence of a chronic carrier state. Some patients with impaired immunity for example, those with renal dialysis or following a transplant do not develop the acute disease but become insidiously ill and eventually undergo progressive liver damage. Lastly, $15 \%$ adults in USA have anti $\mathrm{HB}_{\mathrm{s}}$ without any prior history of exposure or illness, recognized as hepatitis. One of the major concerns in chronic $\mathrm{HB}$ is its relationship with primary hepatocellular carcinoma. The incidence of hepatocellular carcinoma in North America is fairly low but in populations in which the incidence of HB carrier state is extremely high, for example, Africa and Eastern Asia, the incidence of hepatocellular carcinoma is also very high, and is considered as one of the leading causes of death in those areas. Although, there is strong evidence in literature of the development of liver cancer in patients with chronic HB infection, the exact role of hepatitis virus is still speculative. The two most common mechanisms involved in the pathogenesis are, a direct oncogenic effect of $\mathrm{HB}$ on hepatocytes, secondly an indirect effect through the development of chronic active hepatitis and macronodular cirrhosis. ${ }^{3}$ Recent studies indicate that cell lines derived from hepatomas can synthesize the major polypeptides of $\mathrm{HB}_{\mathrm{s}} \mathrm{Ag} .{ }^{4}$

\section{Non A. Non B. Hepatitis}

The recognition and realization that a significant number of cases presenting an acute viral hepatitis lacked the distinguishing features of the known HA and $\mathrm{HB}$ virus. This disorder in which a causative agent could not be isolated is known as Non A - Non $\mathrm{B}$ hepatitis. Since then, various studies have been done to isolate the causative agent (S) and it is found that probably there are two agents involved which resemble $\mathrm{HBV}$.

Non A, Non B hepatitis accounts of $80-90 \%$ post transfusion hepatitis in the United States. ${ }^{5}$ In sporadic acute hepatitis Non A, Non B comprises $20-25 \%$. Although transfusion of blood products is the major mode of transmission, many cases have been reported in intra-venous drug abuse and following commercial factor VIII concentrate administration. The incubation period varies throughout the world; this variation may be due to the inoculating dose of the virus, the route of inoculation and the geographic region.

Non A, Non B hepatitis is generally a mild disease, more than $50 \%$ of patients are asymptomatic. When symptoms do occur, they resemble HBV disease. A chronic carrier state has also been found. Progression to persistent or chronic active hepatitis has been reported in $20-40 \%$ of cases.
As there are no specific serological tests yet available, diagnosis is arrived at by exclusion of the more common $\mathrm{HA}$ and $\mathrm{HB}$ infection and rare causes, i.e., CMV, EBV, Herpes virus and drug abuse.

\section{Isolation and Precautions}

Patients hospitalized with viral hepatitis have traditionally been placed on two types of precautions enteric and blood. As our understanding of the etiology and mode of transmission of viral hepatitis is becoming more clear, the guidelines for care for hospitalized patients are gradually changing. ${ }^{6}$ More simplified isolation techniques and specific precautions to specific types of hepatitis are being used leading to better patient care.

Patients with virus A hepatitis do not have a prolonged viraemic stage. Therefore, the chances of HAV through direct blood contact are not as great. Recent studies, through the use of immune electronic microscopy and radio-immunoassay techniques, have shown that patients who develop symptoms and are hospitalized, may be excreting very low levels of HAV in their stools. Although there is a possibility of nosocomial transmission of $\mathrm{HAV}$, the probability of this happening is very low. Routine enteric precautions that apply for the rest of the hospital patients are sufficient in HAV patients. Isolation in private rooms is not necessary unless the patient is incontinent of feces.

Patients with acute hepatitis B are infective and pose a major problem of transmission of infection through blood, body fluid, excretions and secretions.

Hepatitis B patients are infective in acute phase of infection and in chronic hepatitis B carrier state when they are positive for $\mathrm{HB}_{\mathrm{e}} \mathrm{Ag}$. The modes of transmission are as follows: (1) overt parentral transmission; (2) percutaneous transmission of infective serum or plasma in the absence of overt needle puncture; (3) contamination of mucosal surfaces by infective serum of plasma; (4) contamination of mucosal surfaces by infective substances e.g. mouth pipetting, accidental splashes, sexual activities, etc. In the management of these patients only blood precautions are necessary, enteric precautions are not warranted as fecal-oral contamination in $\mathrm{HB}$ is non-existent. ${ }^{6}$ Special precautions should be taken while collecting blood specimens. Disposable needles and syringes should be used, whenever possible. Bagging and transportation of infective material like tissues and dressing should be done with extreme caution. The laboratory and cleaning personnel should be alerted to the potential hazard. 


\section{Preventive Measures}

The Center for Disease Control (CDC) in Atlan$\mathrm{ta}$, recommends the following measures:

\section{Hepatitis A Prevention:?}

(1) Education of public toward good personal hygiene, careful hand washing and sanitary disposal of feces, to minimize possible fecaloral transmission.

(2) Disposable needles and syringes should be used. Reusable equipment for parentral use should be properly sterilized and decontaminated.

(3) Travelers to endermic areas may be given prophylactic immune serum globulin (ISG) in the following doses: for an expected exposure of up to three (3) months, $0.02 \mathrm{ml}$ per $\mathrm{kg}$ of body weight in a single dose; for a more prolonged exposure $0.06 \mathrm{ml}$ per $\mathrm{kg}$ should be repeated once every four (4) to six (6) months, if exposure continues. Immuno-prophylaxis should also be considered for all new employees to mental institutions, home for retarded and close contact after exposure to an active case.

There is no vaccine available for active immunization against hepatitis A virus.

\section{Hepatitis B Prevention:}

(1) Strict discipline should be enforced in blood banks. Guidelines for blood donations and administration set by the American Association of Blood Banks should be followed precisely to avoid parentral transmission of the virus.

(2) Surveillance of all cases of post-transfusion hepatitis should be maintained.

(3) Immunoprophylaxis, by ISG is effective to some extent as modest amounts of anti $\mathrm{HB}_{\mathrm{s}}$ are present in ISG. Hepatitis B immune globulin (HBIG) an especially developed globulin preparation, now available, although expensive, is effective against acquired hepatitis through accidental needle stick. HBIG may also be useful in infants of HB positive mothers.

Control measures for Non A, Non B hepatitis are the same as that for HBV. Data on the usefulness of prophylactive ISG are limited but suggest that it may be useful in decreasing the incidence of severity of post-transfusion hepatitis when given prior to transfusion. ${ }^{4}$

\section{Hepatitis B Vaccine}

The development of hepatitis B vaccine marks the beginning of a new era in the prevention of HBV infection worldwide. The vaccine has been marketed in many European countries for a number of years. The United States licensed vaccine, prepared by Merck, Sharp and Dohme (MSD) has been given to over 19,000 persons between 1975 and 1982. On June 24, 1982, the Immunization Practices Advisory Committee (ACIP) recommended the use of hepatitis $B$ vaccine for high risk groups, depending on the geographic areas, life style and risk of exposure. ${ }^{8}$

Hepatitis B vaccine is a non-infectious, formalin inactivated virus unit derived from surface antigen. The vaccine is prepared from "closely monitored population of asymptomatic $\mathrm{HB}_{\mathrm{s}} \mathrm{Ag}$ positive donors." The purification and inactivation involves multiple sequential treatments which not only inactivate infectious hepatitis B virus particles but also many other virus groups.

The cost effectiveness of pre-vaccination screening to determine susceptibility depends on the cost of the vaccine, cost of pre-screening tests and prevalence of immunity. ${ }^{8}$ The immunization regime consists of three (3) doses of vaccine intramuscularly, the first two at one month intervals and the third dose six (6) months after the first dose.

Post vaccine immunity testing has shown that $\mathrm{HBV}$ vaccine produces protective anti-bodies in approximately $90 \%$ of healthy persons.

Despite earlier concerns that $\mathrm{HBV}$ vaccine might carry an etiologic risk for AIDS, to date, no AIDS in vaccine recipients has been reported in outside groups with high AIDS incidence. ${ }^{9}$ CDC, Atlanta has cleared hepatitis vaccine of AIDS transmission.

In summary, this paper highlights the recent advances that have been made in the field of viral hepatitis in the last two decades.

\section{Bibliography}

1. Robbins, S.L. and Cotran, R.S.: Pathological basis of disease, 1979, p. 1935.

2. Hoofnagle, J.H., Perspectives on viral hepatitis: No. 2, 1981, p. 4.

3. Robbins, S.L. and Cotran, R.S.: Pathological basis of disease, 1979, p. 1065-66.

4. Simon, J.B.: Recent advances in viral hepatitis: Annals RCPSC, Vol. 14, No. 5, October 1981 , p. 360.

5. Gitnick, G.: Perspectives on viral hepatitis Non A, Non B hepatitis 1981, No. 1, p. 1.

6. Favero, M.S. and Maynard, J.C.: Guidelines for the care of patients with viral hepatitis; Ann. Int. Med. 1979, 91:872-76.

7. Benson, A.S.: Control of communicable diseases in man; 13 th edition 1980, p. 163-64.

8. ACIP: Inactivated hepatitis B vaccine: Mortality and Morbidity weekly report, 1982, Vol. 31, 317-22 and 327-28.

9. Mortality and Morbidity weekly report: 32 (10) p. 134-36, March 18, 1983.

For reprints: Rasheda Nyeem, M.D. 2019 N. W. 21st Lane

Gainesville, Florida 32605 